\title{
Coffee Leaf Disease Recognition Using Gist Feature
}

\author{
Md. Burhan Uddin Chowdhury \\ Kazem Ali School and College, Chittagong, Bangladesh \\ chowdhuryburhan57@gmail.com
}

Received: 04 February 2021; Accepted: 27 March 2021; Published: 08 April 2021

\begin{abstract}
Coffee leaf disease recognition is important as its quality can be affected by the disease like -rust. This paper presents a coffee leaf disease recognition system with the help of gist feature. This research can help coffee producers in diagnosis of coffee plants in initial stage. Rocole coffee leaf dataset is considered in this study. Input image is preprocessed first. Resize and filtering is used as pre-processing work. Gist feature is extracted from pre-processed image. Extracted features are trained with machine learning algorithm. In testing phase, features are extracted and tested with trained ML model. Simulation is done with 10 fold cross validation. Different ML models are used and selected the best among them based on performance. SVM achieved overall 99.8\% accuracy in recognizing coffee leaf disease.
\end{abstract}

Index Terms: Leaf disease, gist feature, support vector machine

\section{Introduction}

Coffee plant grows all over the world in more or less. People usually have coffee in leisure period. Coffee that are collected from coffee plants may effected by disease. Two terrible diseses are brown eye spot and Rust. Effecting yellow halo surrounding with dark in the center and nercotic lesions rounded with brown color is symptom of brown eye spot disease[1]. This disease reduces growth rate of plant. Leaves started to fall down rapidly. Shapeless lesions is the cause of leaf rust disease. This study focused on leaf rust disease as an unhealthy leaf. Inadequate weather condition, fungus infect, dry season are the main cause of leaf rust disease [2]. Rocole coffee plant dataset is used in this study [15]. The dataset contains healthy class and different rust level classes. This study considered healthy leaf images as a class and all rust level images as unhealthy class. Total 640 images are used for two classes.

Rust disease usually occurred in older coffee plants. So if proper step is not taken in time, there can be a huge loss of productivity and as well as time too. So it is necessary to speed up the process of identification of rust leaf diseases. Machine learning can play a key role in this case. Few solutions of classifying coffee leaf disease are presented earlier based on deep learning[16]. But deep learning consumes much time. Global feature based learning method with higher performance can be a suitable solution for recognizing unhealthy or leaf disease. So using gist along with glcm features based classification of coffee leaf disease is proposed in this study.

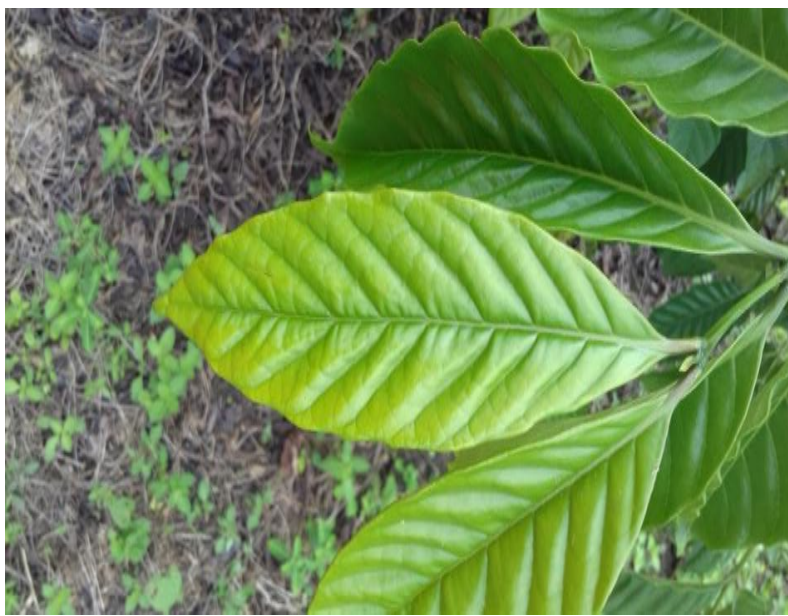

(a)

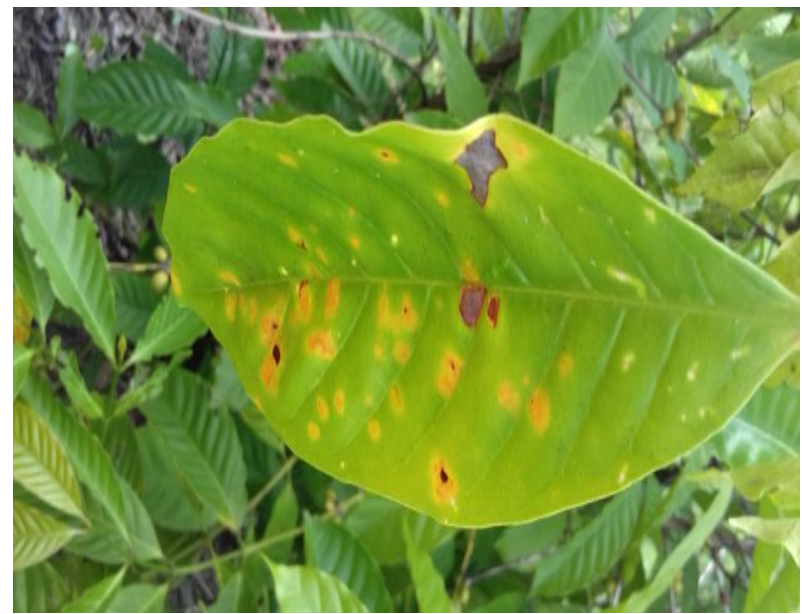

(b)

Fig. 1. (a) Healthy leaf and (b) unhealthy(rust) leaf 
Coffee leaf diseases are shapeless. So content based feature is can perform in a good way. In this context gist feature is considered in this research. Gist feature is popular for scene-based classification [13]. Gist feature with different dimensions is tested. Proposed system recognizes leaf rust disease and healthy leaf based on gist feature. SVM is used as a classifier.

\section{Related Work}

Deep learning has become very popular for classifying objects. Fuentes proposed CNN based method to recognize tomato disease [3]. It includes Faster R-CNN, R-FCN and single shot multi-box detector (SSD).This formation is named as 'deep learning meta-architectures'. Each component of the meta-architectures is combined with deep feature extractors like ResNet or VGG net. Expert system based on fuzzy logic and decision tree is used by Suhartono [4]. Symptoms and disease information is gathered from a human expert. Then rules are set based on the information collected from expert and decision tree is used for classification. Image processing based leaf disease detection method is developed by S.D.A [5]. A robot takes decision to apply pesticide to the leaf. The agri-robo detects disease and spray pesticides to the plants so as to provide protection from disease. The robot also keep monitors the crops.

A method to recognize fungal disease effected leaves is presented by Pujari [6]. The method uses radon transform and SVM. It first identifies whether a cereal is normal or fungal affected. K- means segmentation is used to segment fungal area. After identification, the fungal area is the classified by SVM. Segmentation of the infected area and then classification is done by Gutte [7]. Segmentation is accomplished by k-means clustering and Support vector machine is used for classification. Color, shape and texture features are extracted from the segmented area. Arivazhago presented a system that uses CCM to extract features from native plants [8]. Green pixels are removed followed by thresholding from input image. Then features are extracted from the segmented image .Color and texture features are selected by CCM. Finally SVM is used to classify plants leaf.

Dheeb proposed a framework to detect and classification of leaf diseases [9]. $\mathrm{K}$ means techniques is used for segmentation. HIS color space is considered. Color properties collected as feature and neural network classifier is applied as classifier. Premalatha and Valarmathy proposed system that used both spatial FCM to segment\& PNN (Probabilistic neural network) classifiers to identify cotton plant disease [10]. After applying median filter to the input image. Spatial FCM clustering method is applied to segment the filtered image. PNN classifies with color and texture features. Haiguang presented a method which extracts color, shape and texture to classify grapes leaf [11]. K measn clustering is used for segmenting image. Principle component analysis( PCA) is employed to reduce feature dimension Features are color, shape, texture. . Finally neural network is applied for classification task.

S. Phadikar J. Sil, presented a work to detect rice diseases- leaf brown spot disease and the leaf blast disease. Detection was based on the changes of morphological structure of plants. Classification task performed by Bayes and SVM which provides $68.1 \%$ and $79.5 \%$ accuracy on test images respectively [12]. Megha and Malavika developed a model to classfy tomato leaf disease[17]. Late Blight Disease is considered by them. Thresholding algorithm is proposed by them to classify a leaf as healthy or infected. . K -means clustering algorithm was used to analyze late blight disease. The. Tomato leaf was collected from various plantations.Identification of ornamental plant from its leaf was developed by Kohei [18]. The system utilizes shape and color features. Dyadic Wavelet Transformation Zernike Complex Moment are used for descripting shape and HSV for color descriptions. A recognition system to classify botanical species from image of leaf is developed by Heba and Ashraf [19]. Flavia and swedish dataset were used in their study. The system uses a combined result of different order statistics based measures.

\section{Methodology}

Acquisiton of image is the first stage of a recognition system. For training, a number dataset is supplied as input. Each image is resized and then filtered to remove noise. Filtering causes smoothing of image.It also enhances the qulity of an image. An image may have various effects. So removing of noise makes the image better for further processing. Then comes feature extraction phase. Gist feature is considered in this study. Gist classifies an image as a scene. Dataset have images with background also. For this purpose gist feature is well suitable. So, gist feature is extracted from images. Gist can provides feature in various dimensions. Different dimensional features are extracted. Then Extracted features are sent for training. After completion of training, a trained model is stored which is going to be used for testing. In testing phase, test image is taken and processed for pre-processing. The test image is then resized and filtered. Filtering is for removing noises from image. Then gist feature is extracted. Extracted feature matrix is forwarded to the trained model. The model classifies the test image and shows class label. 


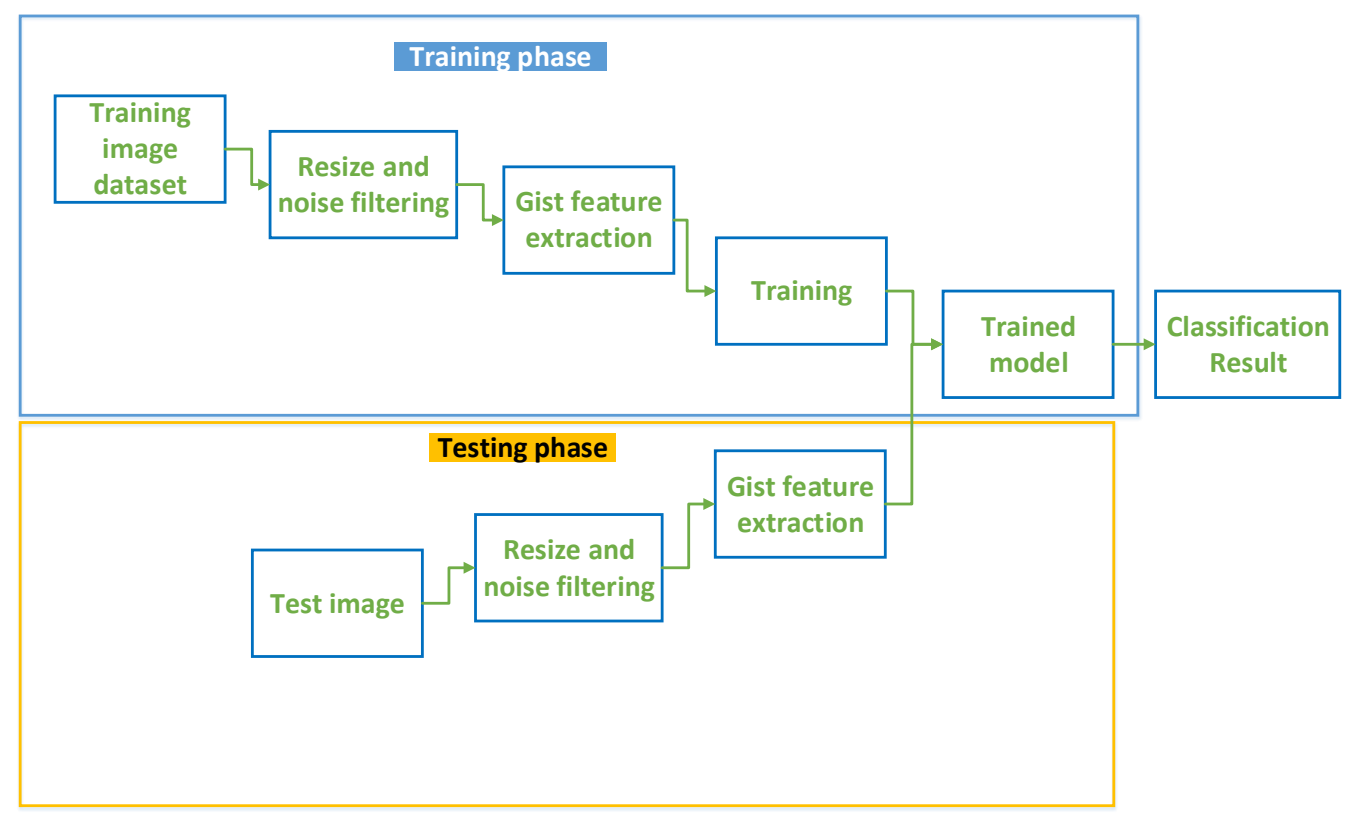

Fig. 2. Block diagram of proposed system

\section{Gist Feature}

The GIST descriptor can describes scene with low dimensional feature matrix. It is also proved that gist descriptor performs good in scene classification [13]. Use of Gist descriptors in applications such as web-scale image search have been proven beneficial in a large variety [14]. Gist descriptors are designed for matching human thoughts with respect to different features of images. Gist descriptor basically provides spatial structure of a scene. Gist is a collection of perceptual dimension.

Gist features can be extracted with different dimensional feature vector. This study focused on extracting gist descriptor in two different dimensions. 128 dimensional and 256 dimensional feature vectors are extracted in this research. These two different dimensional feature vectors trained and tested individually. Both feature dimensions performed approximately equal.
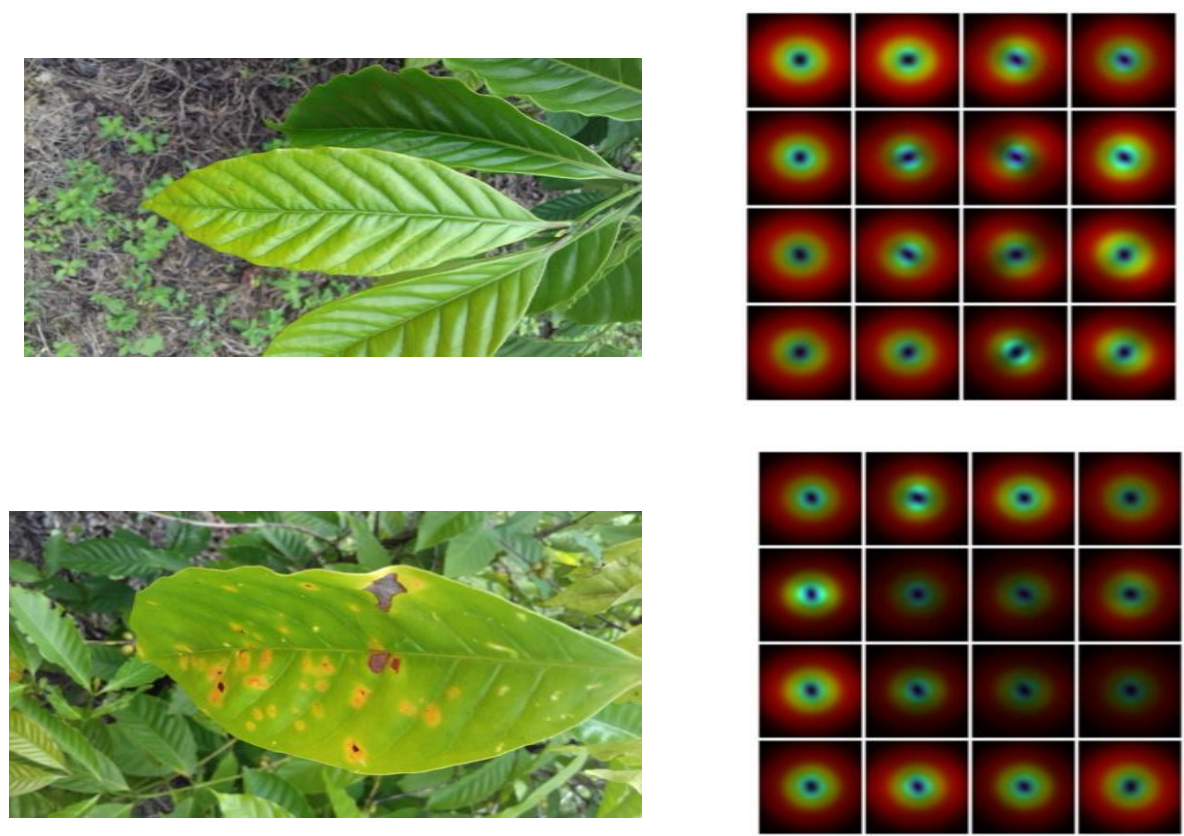

Fig.3. Gist feature visualization 


\section{Performance Analysis}

Confusion matrix and receiver operating characteristic (ROC) curve are usually used to analyze the performance of a classifier. Confusion matrix plots the true positive rate against false positive rate. Table 1 shows the classification result of different classifier with different dimensions. SVM shows better result in compared with others. In both dimension, results are same by the classifier, though adaboost performed slightly different.

Table 1. Result of different classifiers with different dimensions

\begin{tabular}{|c|c|c|}
\cline { 2 - 3 } \multicolumn{1}{c|}{} & \multicolumn{2}{c|}{ Accuracy rate (\%) } \\
\hline Classifier & Dimension size=128 & Dimension size=256 \\
\hline SVM & 99.8 & 99.8 \\
\hline Adaboost & 96.9 & 97.0 \\
\hline Naiive Bayes & 73.3 & 73.3 \\
\hline KNN & 75.8 & 75.8 \\
\hline
\end{tabular}

10 fold cross validation process completes training. One set was used for testing and the remaining for training until 10 iterations. The confusion matrix achieved by SVM classifier is shown in fig. 4. Confusion matrix is basically the summary of prediction. It helps to analyze the types of errors and also correct classifications.

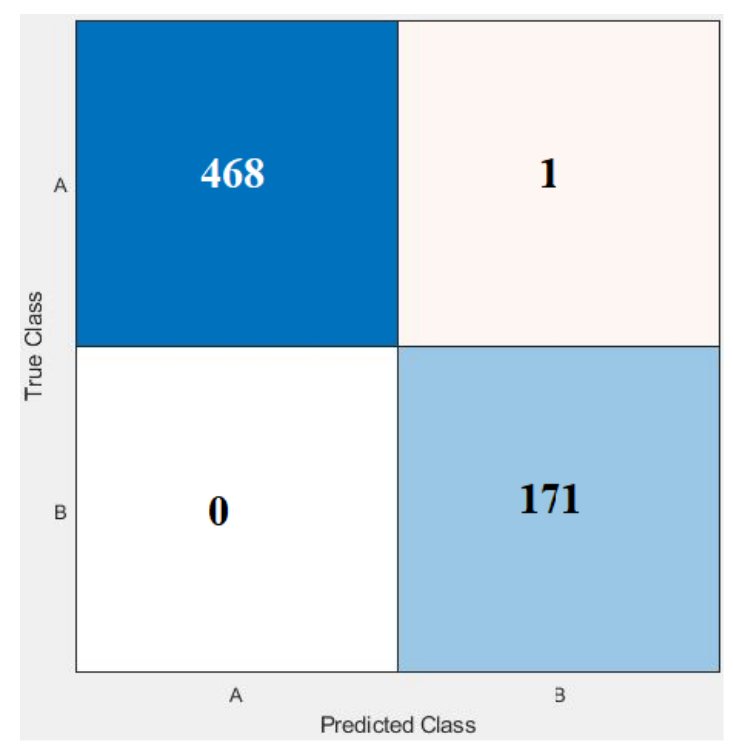

Fig.4. Confusion matrix of SVM classifier

ROC is a graphical illustration that presents true positive rate vs false positive rate. The AUC (Area under curve) for each class is equal to 1 which is notable. ROC curve for each class is presented in fig. 5 and fig. 6 


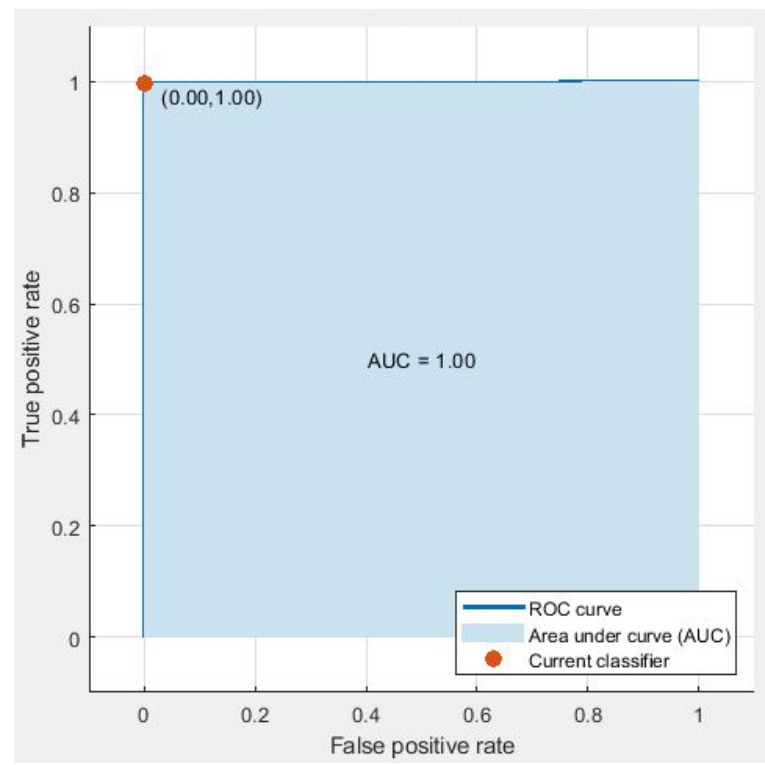

Fig.5. ROC curve of healthy class

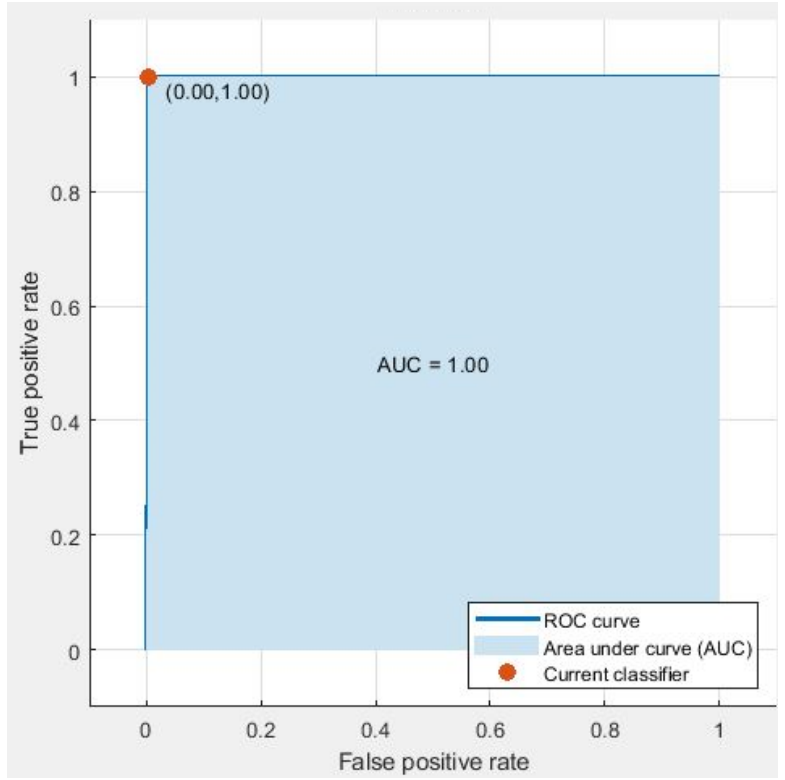

Fig. 6. ROC curve for unhealthy (rust disease) class

Classification result shows precision, recall and f1 measure for each class. Table 2 displays the result of classification report. It also helps to understand and analyze the performance of a classifier.

Table 2. Classification result of SVM

\begin{tabular}{|l|l|l|l|}
\cline { 2 - 4 } \multicolumn{1}{c|}{} & precision (\%) & recall (\%) & f1-score (\%) \\
\hline Healthy & 100 & 99.7 & 99.8 \\
\hline Unhealthy/Rust disease & 99.4 & 100 & 99.6 \\
\hline Accuracy & \multicolumn{3}{|c|}{99.8} \\
\hline
\end{tabular}




\section{Conclusion}

The work presents gist feature based classification of coffee leaf, which can help coffee producers to maintain coffee plants more safely. Also early detection of leaf disease can lead to better treatment and recovery of plants leaf. This paper also helps to investigate the strength of gist feature. Different dimensional feature vectors are also analyzed in this work. Performances is also satisfactory and acceptable. Proposed system is simulated on two different dimensional feature vector and the result is appreciable. Proposed systems shows a good performance. In future, we aim to different pre-processing and post-processing of image. Moreover, new kind of features will be tried out to explore, include more diseases and compared them with others

\section{References}

[1] Pereira, Cassiano Spaziani, Rubens José Guimarães, Edson Ampélio Pozza, and Adriano Alves da Silva. (2008). "Controle de Cercosporiose e da Ferrugem do Cafeeiro com Extrato Etanólico de Própolis" Ceres 55(5): 369-376.

[2] Kushalappa, A.C., M.Akutsu, S.H. Oseguera, G.M.Chaves et al. (1984). "Equations for Predicting the Rate of Coffee Rust Development Based on Net Survival Ratio for Monocyclic Process of Hemileia Vastatrix [Coffea Arabica].” Fitopatologia Brasileira (Brazil)

[3] Fuentes, Alvaro, Sook Yoon, Sang Cheol Kim, Dong Sun Park. (2017). “A Robust Deep-Learning-Based Detector for RealTime Tomato Plant Diseases and Pests Recognition”. Sensors, 17(9), 1-21.

[4] Suhartono, Derwin, Wahyu Aditya, Miranty Lestari, and Muhamad Yasin. (2013). "Expert System in Detecting Coffee Plant Diseases." International Journal of Electrical Energy 1 (3): 156-162..

[5] S. D. A, G. A. G, C. P. A, and K. P. L, “ Intelligent autonomous farming robot with plant disease detection using image processing," International Journal of Advanced Research in Computer and Communication Engineering, vol. 5, no. 4, pp. 1012-1016, 2016

[6] J. D. Pujari, R. Yakkundimath, and A. S. Byadgi, "Detection and classification of fungal disease with radon transform and support vector machine affected on cereals," Int. J. of Computational Vision and Robotics, vol. 4, pp. 261-280, 2014.

[7] Gutte, Vitthal S., Maharudra A.Gitte. (2018). "A Survey on Recognition of Plant Disease with Help of Algorithm". International Journal of Engineering Science and Computing, 6(6), 7100-7102.

[8] Selvaraj, Arivazhagan \& Shebiah, Newlin \& Ananthi, S. \& Varthini, S.. (2013), "Detection of unhealthy region of plant leaves and classification of plant leaf diseases using texture features", Agricultural Engineering International: CIGR Journal. 15. 211217

[9] Dheeb Albashish \& Malik Braik \& Sulieman Bani-Ahmad (2011). A framework for detection and classification of plant leaf and stem diseases. 113 - 118. 10.1109/ICSIP.2010.5697452.

[10] V. Premalatha, S. Valarmathy and M. G. Sumithra, "Disease Identification in Cotton Plants Using Spatial FCM \& PNN Classifier", vol. 3, no. 4, (2015).

[11] H. Wang, G. Li, Z. Ma and X. Li, "Image Recognition of Plant Diseases Based on Back propagation Networks", IEEE, (2015)

[12] S. Phadikar, J. Sil and A. K. Das, "Classification of Rice Leaf Diseases Based on morphological Changes", Breeding Science, (2008), pp. 93-96.

[13] A. Oliva and A. Torralba, "Modeling the shape of the scene: A holistic representation of the spatial envelope," Int. J. Comput. Vision, vol. 42, pp. 145-175, May 2001.

[14] Douze, M., Jegou, H., Sandhawalia, H., Amsaleg, L., and Schmid, C. (2009). Evaluation of gist descriptors for web-scale image search. In Proceedings of the ACM International Conference on Image and Video Retrieval, CIVR '09, pages 19:1-19:8, New York, NY, USA. ACM

[15] Parraga-Alava, Jorge; Cusme, Kevin; Loor, Angélica; Santander, Esneider (2019), "RoCoLe: A robusta coffee leaf images dataset ", Mendeley Data, V2, doi: 10.17632/c5yvn32dzg.2

[16] A. P. Marcos, N. L. Silva Rodovalho and A. R. Backes, "Coffee Leaf Rust Detection Using Convolutional Neural Network," 2019 XV Workshop de Visão Computacional (WVC), São Bernardo do Campo, Brazil, 2019, pp. 38-42, doi: 10.1109/WVC.2019.8876931.

[17] Megha P Arakeri, Malavika Arun, Padmini R K,"Analysis of Late Blight Disease in Tomato Leaf Using Image Processing Techniques", International Journal of Engineering and Manufacturing(IJEM), Vol.5, No.4, pp.12-22, 2015.DOI: 10.5815/ijem.2015.04.02

[18] Kohei Arai, Indra Nugraha Abdullah, Hiroshi Okumura"Image Identification Based on Shape and Color Descriptors and Its Application to Ornamental Leaf", IJIGSP, vol.5, no.10, pp.1-8, 2013.DOI: 10.5815/ijigsp.2013.10.01

[19] Heba F. Eid, Ashraf Darwish, "Variant-Order Statistics based Model for Real-Time Plant Species Recognition", International Journal of Information Technology and Computer Science (IJITCS), Vol.9, No.9, pp. 77-84, 2017. DOI: 10.5815/ijitcs.2017.09.08 


\section{Author's Profile}

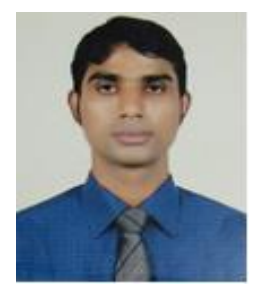

Md. Burhan Uddin Chowdhury received the B.Sc. degree in Computer and Communication Engineering from International Islamic University Chittagong, Bangladesh and M.Sc. degree in Computer Science \& Engineering from Port City International University, Bangladesh. Currently he is working as a lecturer in ICT at Kazem Ali School and College, Chittagong, Bangladesh. His research interest includes pattern recognition and signal processing.

How to cite this paper: Md. Burhan Uddin Chowdhury, " Coffee Leaf Disease Recognition Using Gist Feature", International Journal of Information Engineering and Electronic Business(IJIEEB), Vol.13, No.2, pp. 55-61, 2021. DOI: 10.5815/ijieeb.2021.02.05 